\title{
SUCCESSFUL TREATMENT OF LIFE THREATENING INTRACRANIAL AVM BY ENDOVASCULAR EMBOLIZATION IN DHAKA MEDICAL COLLEGE HOSPITAL
}

\author{
SHARIF UDDIN KHAN ${ }^{1}$, KAZI MOHIBUR RAHMAN ${ }^{1}$, MD. MAHMUDUR RAHMAN SIDDIQUI ${ }^{2}$, MD. \\ AZHARUL HOQUE ${ }^{3}$, MD. BADRUL ALAM MONDOL ${ }^{3}$, SHAKIR HUSAIN $^{4}$, QUAZI DEEN MOHAMMAD ${ }^{5}$
}

\begin{abstract}
Arteriovenous malformations (AVMs) are tangled, dilated blood vessels in which arteries flow directly into veins. AVMs can bleed or directly compress brain tissue; seizures or ischemia may result. Haemorrhagic stroke from AVM represents $2 \%$ of all haemorrhagic strokes. A clear understanding of the diagnostic and treatment algorithms of cerebral AVM management is very important because AVMs are a cause of hemorrhage in young adults. We report a 16 years old boy with cerebral arteriovenous malformation (AVM), complicated by recurrent throbbing headache, vomiting and seizure. This was clearly diagnosed by Digital subtraction angiogram (DSA). He was successfully treated by endovascular embolization at Dhaka Medical College Hospital. This was the second successful cerebral endovascular embolization in any government hospital of Bangladesh.
\end{abstract}

Keyword: Arteriovenous malformation, seizure, digital subtraction angiogram, endovascular embolization.

\section{Introduction}

Intracranial arteriovenous malformations (AVMs) are complex tangled, dilated blood vessels in which arteries flow directly into veins without capillaries. ${ }^{1}$ AVMs occur most often at the junction of cerebral arteries, usually within the parenchyma of the frontal-parietal region, frontal lobe, lateral cerebellum, or overlying occipital lobe. AVMs can bleed or directly compress brain tissue; seizures or ischemia may result. ${ }^{1}$ The main cause of death in patients with cerebral arteriovenous malformations $(\mathrm{AVM})$ is intraparenchymal hemorrhage. ${ }^{1}$ This clinical event represents the first symptom in about $30-55 \%$ of reported cases, with an annual incidence of 2-3 \%. ${ }^{1}$ AVMs cause about $2 \%$ of all hemorrhagic strokes each year, but in our country we do not have any statistical data about cerebral AVMs. ${ }^{2}$ Here we report a case of cerebral arteriovenous malformation (AVM), complicated by recurrent headache and seizure. This was successfully treated with endovascular embolization.

\section{Case Report}

A 16 years old boy was admitted in department of Neurology, Dhaka Medical College Hospital (DMCH) with the complaints of occasional headache associated with tingling sensation and numbness of the right forehead followed by vomiting and decreased level of consciousness for last 7 years. He also developed 2 episodes of focal convulsions within last 1 year.

1. Assistant Professor, Department of Neurology, Dhaka Medical College Hospital, Dhaka, Bangladesh.

2. Postgraduate Resident, Department of Medicine, Dhaka Medical College Hospital, Dhaka, Bangladesh.

3. Associate Professor, Department of Neurology, Dhaka Medical College Hospital, Dhaka, Bangladesh.

4. Chief Neuroendovascular and Stroke therapy Department, Sir Ganga Ram Hospital, New Deilhi, India.

5. Professor and Head, Department of Neurology and Principal, Dhaka Medical College, Dhaka, Bangladesh.

Correspondence: Dr. Sharif Uddin Khan, Assistant Professor, Department of Neurology, Dhaka Medical College Hospital, Dhaka, Bangladesh. E-mail: sharif.911@gmail.com 
Clinical examination on admission revealed patient is well oriented, pulse $80 \mathrm{~b} / \mathrm{min}$, blood pressure $110 /$ $70 \mathrm{~mm}$ of $\mathrm{Hg}$. All systemic examinations revealed normal.

All baseline investigations were normal but Magnetic Resonant Imaging (MRI) of brain revealed serpigenous signal void areas were noted in right temporo-parietal region (fig 1). After intra-venous contrast administration heterogeneous enhancement of the lesion area was noted in same region. These features were compatible with arteriovenous malformation (AVM). Magnetic Resonant Angiogram (MRA) of brain showed right parietal AVM supplied by right Middle Cerebral Artery (MCA) (fig 2). He also underwent cerebral Digital Subtraction Angiogram (DSA) for detailed information about AVM, which revealed right sided medium to large sized high flow parietal AVM supplied by right Middle Cerebral Artery (MCA) with ectatic cortical vein.

For therapeutic purpose micro catheterization of callosal and pericallosal branch of right ACA and right MCA was done and AVM was embolized with PVA (poly vinyl alcohol). After embolization, check cerebral DSA showed complete disappearance of the AVM (fig 3). Post procedure period was uneventful. After discharge, he was symptom free and still leaving a healthy life.

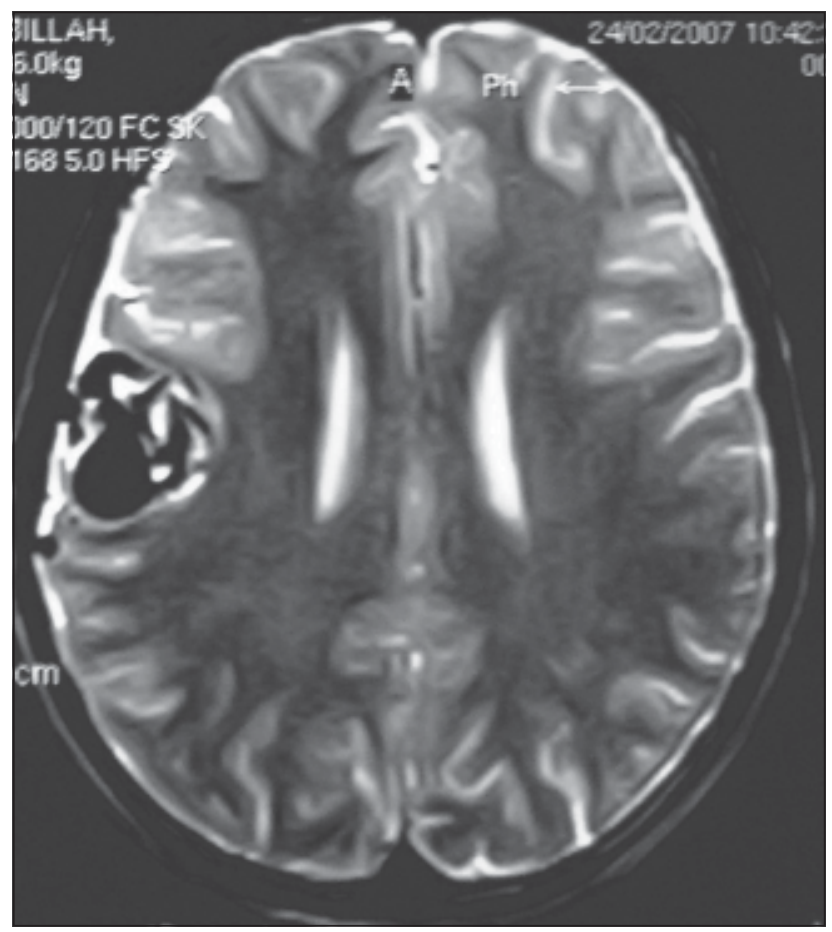

Fig.-1: T2 weighted MRI of brain shows serpigenous signal void areas in right temporo-parietal region (arrow).

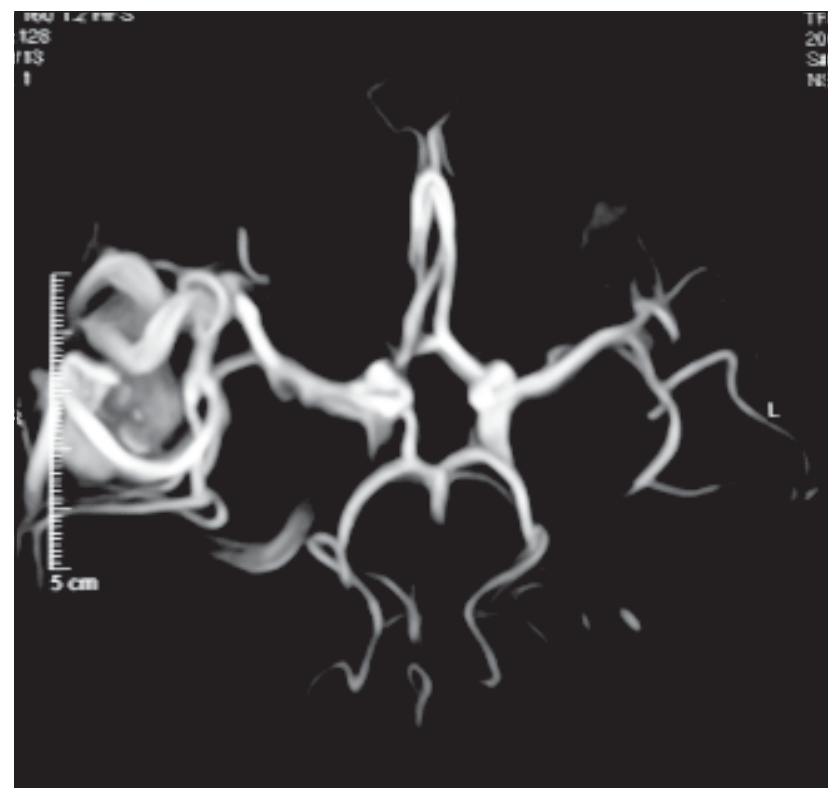

Fig.-2: MRA of brain shows right parietal AVM supplied by Right MCA (arrow).

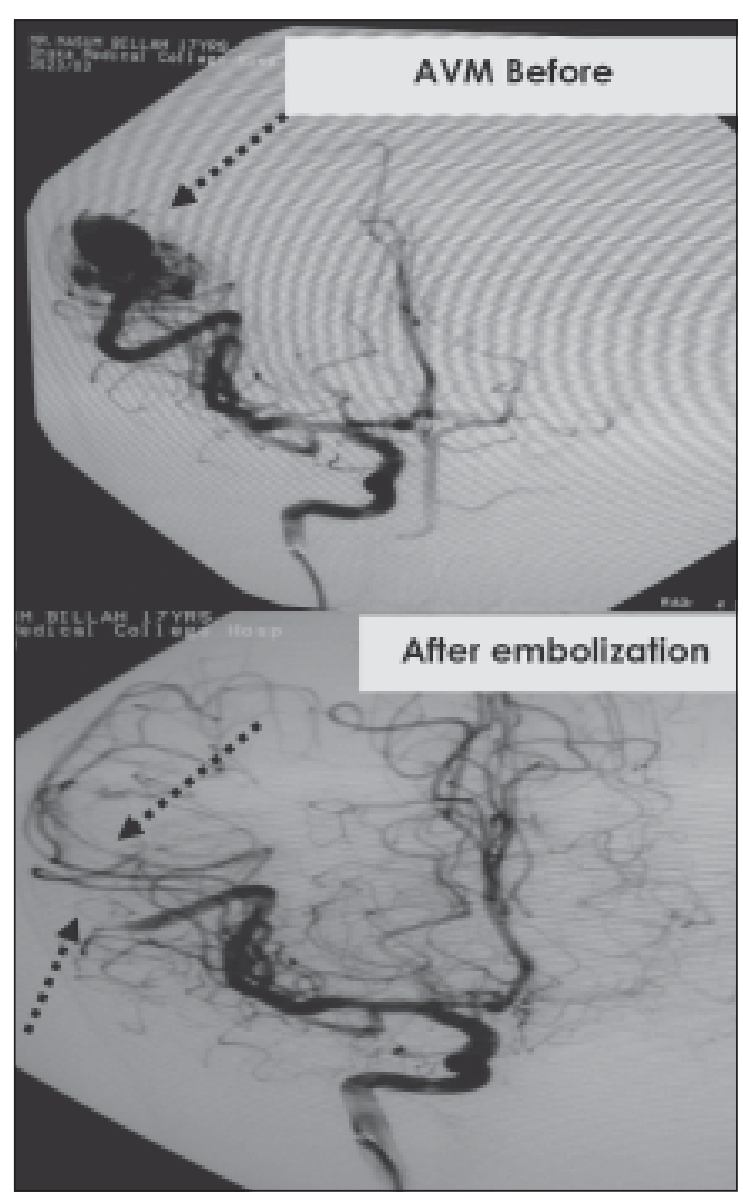

Fig.-3: Cerebral DSA shows a large AVM in right MCA branches which completely disappears after embolization (arrows). 


\section{Discussion}

The vascular conglomerate of an AVM is called the nidus. The nidus has no capillary bed, and the feeding arteries drain directly to the draining veins. AVMs of the brain are congenital and relatively rare. Approximately 300,000 (0.14\%) Americans have AVMs, but only $12 \%$ of them experience symptoms. ${ }^{3}$ They affect both men and women at same rate, but most often cause symptoms between 20 and 40 years of age. 3 They frequently occur as isolated, stable anomalies requiring no specific treatment. However, some AVMs are extensive and progressive, having the potential for life-threatening hemorrhage, thrombosis. ${ }^{4,5} \mathrm{AVMs}$ produce neurological dysfunction through hemorrhage in the subarachnoid space, intraventricular space or, most commonly in the brain parenchyma. Generalized or focal seizures may present in $30 \%$ and $10 \%$ cases respectively. ${ }^{3}$ Neurological deficits can also occur by the mass effect of an enlarging AVM or venous hypertension in the draining veins.$^{2,3,4,5}$

Computer Tomography (CT) scanning easily identifies an intracerebral hemorrhage. In Magnetic resonance imaging (MRI) AVMs appear as irregular or globoid masses. Small, round, low-signal spots within or around the mass on T1, T2, or fluid-attenuated inversion recovery (FLAIR) weighted images are the "flow voids" of feeding arteries, intranidal aneurysms, or draining veins. ${ }^{6}$ Magnetic resonance angiography (MRA) may identify AVMs greater than $1 \mathrm{~cm}$ in size, but is inadequate to identified the morphology of feeding arteries and draining veins; small aneurysms can be missed easily. ${ }^{3,6}$ So, now a day cerebral digital subtraction angiogram (DSA) is the gold standard investigation to find out the detail information about AVMs. ${ }^{6}$ In this case, we also got the actual picture of AVMs with the help of DSA.

The aim of modern invasive treatment of AVM is to eliminate the source of hemorrhage, comprising microsurgical resection, endovascular embolization, and radiotherapy, alone or in combination. Embolization of high-grade intracranial arteriovenous malformations (AVMs) before surgical resection is an accepted treatment technique to improve the outcome. ${ }^{2,4}$ The goal of embolization before surgery or radiosurgery is to reduce the AVM volume and to occlude critical feeders. Surgical resection alone can be done without primary embolization in low-grade AVMs. AVMs in anatomically difficult locations can be treated by Radiosurgery. ${ }^{7}$ Many endovascular embolic agents are currently available for treating AVMs, including autologous clot, Gelfoam, polyvinyl alcohol (PVA) particles, stainless steel or titanium coils, acrylic tissue adhesives, detachable balloons, and liquid sclerosing agents. ${ }^{8,9}$ Due to the advent of superselective angiographic catheters that allow selective embolization of the AVM sources, embolization therapy has tended to replace surgery as the treatment of choice for this disorder. Our case was also successfully treated by endovascular embolization with PVA particles.

\section{Conclusion}

Brain AVMs can cause cerebral hemorrhages, with devastating consequences for the patients and their families. Cerebral digital subtraction angiogram (DSA) followed by therapeutic intervention like neuro-endovascular stenting, embolization of AVM by PVA particle is now a day considered as unique method of treatment. Therapeutic neurointervention in treating such vascular neurological diseases is an established method of treatment in Dhaka Medical College Hospital.

\section{References}

1. Puzzilli F, Mastronardi L, Ruggeri A, et al. Early surgical treatment of intracerebral hemorrhages caused by AVM: our experience in 10 cases. Neurosurg. Rev 1998;21:87-92.

2. Jae HC, Henning M, Robert RS, et al. Clinical Outcome After First and Recurrent Hemorrhage in Patients With Untreated Brain Arteriovenous Malformation. Stroke 2006;37:1243-1247.

3. Hofmeister C, Stapf C, Hartmann A, et al. Demographic, Morphological, and Clinical Characteristics of 1289 Patients With Brain Arteriovenous Malformation. Stroke 2000;31:1307-1310.

4. Haas PC, Angelini P, Leachman R, et al. Percutaneous Treatment of Life Threatening Congenital Arteriovenous Malformations With the Wallgraft Endoprosthesis. J Endovasc Ther 2000;7:333-339.

5. Mast H, Mohr JP, Osipov A, et al. "Steal" is an unestablished mechanism for the clinical presentation of cerebral arteriovenous malformations. Stroke1995; 26:1213-1220.

6. Christian SH, Randolph SM. Arteriovenous Malformations. Available at http://emedicine. medscape.com/article/1160167-overview.

7. Weber W, Kis B, Siekmann R, et al. Endovascular Treatment of Intracranial Arteriovenous Malformations with Onyx: Technical Aspects; AJNR Am J Neuroradiol 2007; 28:371-77.

8. Swarc TA, Carrasco CH, Wallace S, et al. Radiopaque suspension of polyvinyl alcohol foam for embolization. Am J Roentgenol 1986;146:591-592.

9. Rao VR, Mandalam KR, Gupta AK, et al. Dissolution of isobutyl 2-cyanoacrylate on longterm follow-up. Am J Neuroradiol 1989;10:135-141. 